\section{P267 ASSESSMENT OF COGNITIVE DYSFUNCTION USING THE COGNITIVE FAILURES QUESTIONNAIRE (CFO) TOOL IN PATIENTS WITH OBSTRUCTIVE SLEEP APNOEA SYNDROME (OSAS)}

doi:10.1136/thoraxjnl-2012-202678.359

'A Dwarakanath, 'D Ghosh, ${ }^{2} \mathrm{~S}$ L Jamson, 'M W Elliott. 'St James' University Hospital, Leeds, Leeds, UK; ${ }^{2}$ Institute for Transport Studies, University of Leeds, Leeds, UK

Introduction A wide range of cognitive deficits has been identified in patients with untreated OSAS and there has been a growing interest in the evaluation of cognitive deteriorations. The CFO (Cognitive Failures Questionnaire) is a measure of self-reported deficits in the completion of simple everyday tasks that a person should normally be capable of completing without error and includes failures in attention, memory, perception, and motor functions. We hypothesised that patients with OSAS exhibit worse cognitive dysfunction as compared to healthy controls and this can be evaluated with the CFO.

Methods 133 (ESS $12+/-6$, ODI $31+/-24$ ) untreated OSAS patients and 50 healthy controls (ESS $3+/-2$ ) were invited to complete the CFO. Patients and controls were asked how often they make various common mistakes on a 5-point Likert scale, from 0 (never) to 4 (very often). CFQ was scored by adding up the ratings for twenty-five questions, the highest possible total being one hundred, with a higher score indicating a higher incidence of cognitive failures. Comparisons were made using one way ANOVA.

Results There was a significant difference in scores between patients and the controls $(26 \pm 1.6 \& 38 \pm 1.6, \mathrm{P}<0.0001)$. This difference remained significant when different severities of OSAS (mild, moderate, severe, as per ODI) were compared with controls. However there was no difference when different severities of OSAS were compared against each other (Figure 1).

Conclusion Patients with OSAS have a significant higher CFO score compared to healthy controls. The CFQ is easy to complete and score and our data suggests it may be a useful tool for assessing cognitive dysfunction in OSAS.

\section{Comparing CFQ Score between controls and patients}

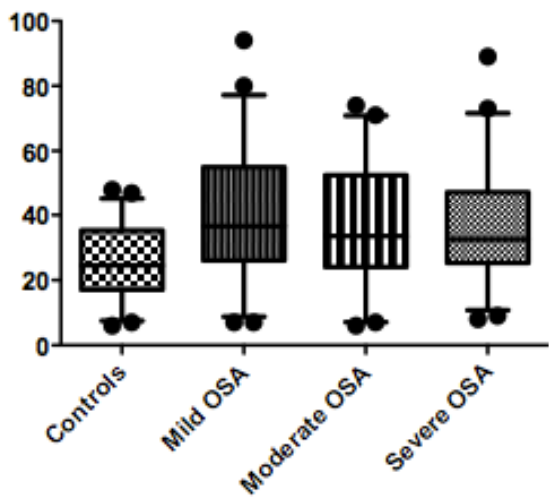

Abstract P267 Figure 1

\section{P268 EFFECT OF CPAP TREATMENT FOR OSA ON VISUAL PROCESSING OF DEGRADED WORDS}

doi:10.1136/thoraxjnl-2012-202678.360

K Proudlove, R Hall, R Rieu, M Villarroel, J Stradling. Oxford Centre for Respiratory Medicine, Churchill Hospital, Oxford, UK

Introduction It has been shown in a 6 month non-randomised clinical trial that patients with both diabetic macula oedema and obstructive sleep apnoea (OSA) improved vision by one line on the $\log$ MAR chart if they used CPAP for more than 2.5 hours/night, compared to those using for less than 2.5 hours/night. However there are many potential explanations for this improvement, including reduced sleepiness leading to increased effort to read the $\log M A R$ chart, rather than any direct improvement in retinal function. We are assessing if non-visually impaired patients, treated for OSA, also improve their ability to read visually degraded words, thus simulating impaired vision.

Methods A specially designed computer programme repeatedly presents five-letter words for three seconds, with one second intervals between. Progressively, the bottom halves of the letters making up the words are removed, making them harder and harder to read. The point at which only $50 \%$ of the words are correctly recognised is 'hunted' by increasing and decreasing the \% revealed, analogous to a hearing test that hunts the auditory threshold. Preliminary tests on normal subjects established that a 10 minute training period, followed by a 10 minute rest, and then by a 10 minute trial period, produced stable results with little or no learning effect over several weeks. 23 symptomatic patients with OSA have been tested pre CPAP, and so far only 5 have been re-tested approximately 2 weeks post CPAP.

Results The average \% of words needing to be revealed to allow $50 \%$ correct recognition in the 23 subjects was $35 \%$ (range 23 to $64 \%$ ). In the 5 restudied so far this was $37 \%$ pre CPAP, and $40 \%$ post. Conclusions Although the study is not yet finished, the preliminary results are not supporting the hypothesis that improved vision in patients with diabetic macular oedema post CPAP treatment is likely to be due to reduced sleepiness, improved vigilance or improved enthusiasm for reading the logMAR chart.

\section{TССт IX $/ \cap D \mathrm{n}$}

\section{CPAP THERAPY DOES NOT REDUCE SERUM URIC ACID LEVELS IN OBSTRUCTIVE SLEEP APNOEA}

doi:10.1136/thoraxjnl-2012-202678.36

'B Prudon, ${ }^{2} E$ Roddy, ${ }^{3} J R$ Stradling, 'SD West. 'Newcastle Regional Sleep Service, Newcastle upon Tyne Hospitals NHS Foundation Trust, Newcastle upon Tyne, UK; ${ }^{2}$ Arthritis Research UK Primary Care Centre, Keele University, Keele, UK; ${ }^{3}$ Oxford Centre for Respiratory Medicine, Churchill Hospital Oxford, Oxford, UK

Obstructive Sleep Apnoea (OSA) is associated with a high prevalence of hyperuricaemia, which occurs in up to $57 \%$ of men with severe OSA, most likely due to increased oxidative stress. Previous work has shown that treatment of OSA with Continuous Positive Airway Pressure (CPAP) reduces serum and urinary uric acid levels, but these studies have been conducted without appropriate control groups. We aimed to assess this effect in a randomised controlled trial.

Stored serum samples from a previously described trial group were analysed (1). This consisted of samples taken from 38 men with known type 2 diabetes and newly diagnosed OSA ( $>10$ dips $/ \mathrm{h}$ $>4 \%$ in oxygen saturation (ODI)), randomised to receive either therapeutic $(n=19)$ or placebo CPAP $(n=19)$ for 3 months. Serum Uric Acid (SUA) levels were measured from samples taken at baseline and after 3 months CPAP. 
Results are expressed as mean (SD). Both groups were well matched at baseline, with no significant difference between age 58.1 years (10.6) therapeutic CPAP group vs. 55.6 (6.9) placebo CPAP group, BMI $36.4 \mathrm{~kg} / \mathrm{m}^{2}$ (5.0) vs. 35.6 (3.6), HbA1c 8.5\% (1.8) vs. $8.1 \%$ (1.6), or ODI $33.9 /$ hour (21.9) vs. $35.7 /$ hour (21.1). There was also no significant difference in therapeutic or placebo CPAP usage $3.7 \mathrm{~h} /$ night (3.2) vs. 3.7 (2.6) $\mathrm{p}=0.8$. There was no significant difference in SUA levels at baseline $362 \mu \mathrm{mol} / \mathrm{l}$ (96) vs. $413 \mu \mathrm{mol} / \mathrm{l}$ (91), or at 3 months $354 \mu \mathrm{mol} / 1$ (83) vs. $406 \mu \mathrm{mol} / 1$ (101). Baseline SUA did not correlate with Apnoea-Hypopnoea Index $(\mathrm{r}=-0.2$, $\mathrm{p}=0.5)$, ODI $(r=0.1, \mathrm{p}=0.6)$, BMI $(\mathrm{r}=0.1, \mathrm{p}=0.4)$, or $\mathrm{HbA} 1 \mathrm{c}$ $(r=-0.3, p=0.9)$. The mean change in SUA at 3 months did not differ significantly between treatment groups $(-7.6 \mu \mathrm{mol} / 1$ (35.9) vs. $-6.2 \mu \mathrm{mol} / 1$ (46.2); $\mathrm{p}=0.9,95 \% \mathrm{CI}-28.7$ to 25.9$)$.

This RCT using therapeutic and placebo CPAP has shown no evidence of a significant reduction in serum uric acid following three months treatment. This is in contrast to previously published uncontrolled data. This study was not however powered to detect a difference in SUA levels and may be therefore underpowered. Further RCTs are needed to explore this effect further.

1. West SD, et al. Thorax. 2007; 62:969-974.

\section{Asthma outcomes}

\section{P270 REDUCING ASTHMA ADMISSIONS BY IMPROVING ASTHMA MANAGEMENT}

doi:10.1136/thoraxjnl-2012-202678.362

1J Gibbs, ${ }^{2} \mathrm{~A}$ Hardwell, ${ }^{2} \mathrm{~A}$ Eagling. 'Medicines Management Department, NHS Bristol, Bristol, United Kingdom; ${ }^{2}$ National Services for Health Improvement, Dartford, Kent, United Kingdom

Introduction There were 79,794 emergency hospital admissions for asthma in the UK in 2008-09 - an estimated 75\% were avoidable [1]. Asthma admissions from Bristol GP practises contributed to this; over 600 people were admitted to hospital in this year because of their asthma. NHS Bristol set out to improve asthma management and reduce asthma hospital admissions across the Primary Care Trust

Method Previous audits identified variations in asthma management across Bristol GP practises. Initial data searches identified some practises provided annual asthma reviews to $73.3 \%$ of their asthma register, others only $36.4 \%$. Inhaler technique was checked in $60.8 \%$ of patients in some practises, in others only $16.9 \%$. NHS Bristol commissioned NSHI Ltd* to run the IMPACT* service across Bristol GP practises to reduce these variations.

The IMPACT service provided a therapeutic review, modular education and detailed clinical review by diploma trained asthma nurses, according to agreed practise protocols. Asthmatic patients were invited to attend a structured clinical review of their asthma. Patients were given a self-management plan and educated in better managing their condition. Practise staff were also provided with enhanced respiratory training.

Results There has been a $19.5 \%$ reduction in asthma admissions in the period from February 2011-January 2012 in Bristol $(n=37)$ compared to the previous year. There were 90 admissions in IMPACT practises $(\mathrm{n}=13)$ and 303 admissions in non-IMPACT practises $(n=43)$ during this period. The IMPACT practises have observed a reduction of $35.6 \%$, and the non-IMPACT practises have observed a reduction of $12.4 \%$. Overall, 13 IMPACT practises accounted for $54.7 \%$ of the reduction.

Conclusion A structured approach to asthma management (including the provision of education to health care professionals in line with national asthma guidelines) and increasing patient awareness of asthma (including knowledge of how to manage their symptoms) can reduce variations in asthma care and hospital admissions.

\section{Reference}

1. Asthma UK for Journalists: Key facts and stats: www.asthma. uk.org (Viewed July 2012).

*NSHI Ltd (National Services for Health Improvement).

*Improving the Management of Patients Asthma and COPD Treatment. (IMPACT) is an independent nurse service sponsored by TEVA UK Limited.

\section{P271 IMPLEMENTING AN ACUTE ASTHMA CARE BUNDLE}

doi:10.1136/thoraxjnl-2012-202678.363

J E McCreanor, J Pollington, T Stocks, L Chandler. Pinderfields General Hospital, Mid Yorkshire NHS Trust, Wakefiled, UK

Background In 2010/11 NHS Wakefield District had the highest rate of admissions for acute exacerbation, in adults, within Yorkshire and Humber. The 2009 BTS adult asthma audit highlighted readmission rates within one month to be $19 \%$ compared to the national average of $8 \%$. Furthermore, the same audit demonstrated that Pinderfields and Pontefract hospitals (part of Mid Yorkshire NHS Trust) were markedly underperforming, compared to national average, in terms of asthma review, patient education and follow up according to BTS/SIGN guidance.

Aims and objectives As part of a new asthma service we introduced an adult acute asthma 'care bundle' to improve the frequency of asthma review, patient education and improve post admission follow up. We also aimed to reduce 28 day readmission rates by $20 \%$ compared to $2010 / 11$

Methods In February 2011 Pinderfields General Hospital merged its acute inpatient medical services with Pontefract General Infirmary enabling a restructuring of the respiratory team and creation of a Mid Yorskhire Asthma service. Following a programme of staff education the acute asthma care bundle was introduced for all adult patients attending the Emergency Department and Acute Medical Unit with an exacerbation of asthma. Data were collected prospectively following the introduction of the care bundle and is compared against the 2009 BTS audit data. Data presented is from the first 46 patients.

Outcomes Following the introduction of the care bundle, inhaler technique assessment was performed in $75.6 \%$ of patients, compared to $38 \%$ previously. Asthma reviews (including self management plan and asthma education) were performed in $88.9 \%$ of patients, compared to $16 \%$ previously. Patients were recommended primary care follow up and had arranged secondary care follow up in 80 and $93.3 \%$ of admissions, compared to 19 and $37 \%$ previously. Furthermore, compared to $2010 / 11$ there was a $66 \%$ reduction in 28 day readmissions, mean monthly average reduced from 5.0 to 1.67 .

Conclusion As part of a restructured respiratory and asthma service the introduction of an acute asthma care bundle led to marked improvements of patient management and 28 day readmission rates.

\section{P272 INFLUENCE OF BMI ON ASTHMA CONTROL OUESTIONNAIRE SCORES}

doi:10.1136/thoraxjnl-2012-202678.364

SZ Zaidi, SJ Jain, HN Nathon, LH Hughes, JF Finnerty, AP Ponnuswamy, IB Benton, SS Scott. Countess Of Chester Hospital, Chester, England

Background Asthma has been associated with obesity. However the mechanisms of this association are not yet clear. It has been suggested that quality of life is influenced more strongly by BMI rather than other objective measures of severity in an obese population 1. We wished toexplore the influence of BMI on asthma control 